\title{
Radio afterglows of binary neutron star mergers: a population study for current and future gravitational wave observing runs
}

\author{
R. Duque, F. Daigne, and R. Mochkovitch
}

\begin{abstract}
Sorbonne Université, CNRS, UMR 7095, Institut d'Astrophysique de Paris, 98 bis boulevard Arago, 75014 Paris, France e-mail: duque@iap.fr
\end{abstract}

Received 20 May 2019 / Accepted 29 August 2019

\begin{abstract}
Following the historical observations of GW170817 and its multi-wavelength afterglow, more radio afterglows from neutron star mergers are expected in the future as counterparts to gravitational wave inspiral signals. Our aim is to describe these events using our current knowledge of the population of neutron star mergers based on gamma-ray burst science, and taking into account the sensitivities of current and future gravitational wave and radio detectors. We combined analytical models for the merger gravitational wave and radio afterglow signals to a population model prescribing the energetics, circum-merger density and other relevant parameters of the mergers. We reported the expected distributions of observables (distance, orientation, afterglow peak time and flux, etc.) for future events and studied how these can be used to further probe the population of binary neutron stars, their mergers and related outflows during future observing campaigns. In the case of the $\mathrm{O} 3$ run of the LIGO-Virgo Collaboration, the radio afterglow of one third of gravitational-wave-detected mergers should be detectable (and detected if the source is localized thanks to the kilonova counterpart) by the Very Large Array. Furthermore, these events should have viewing angles similar to that of GW170817. These findings confirm the radio afterglow as a powerful insight into these events, although some key afterglow-related techniques, such as very long baseline interferometry imaging of the merger remnant, may no longer be feasible as the gravitational wave horizon increases.
\end{abstract}

Key words. methods: statistical - stars: neutron - gravitational waves $-\gamma$-ray burst: individual: GRB170817A $\gamma$-ray burst: general

\section{Introduction}

The first detection of the gravitational waves (GW) from the inspiral phase of a binary neutron star merger (Abbott et al. $2017,2019)$ was followed by all three electromagnetic counterparts expected after the coalescence: a short gamma-ray burst (GRB; Goldstein et al. 2017; Savchenko et al. 2017) and its multi-wavelength afterglow (AG) in the X-ray (Haggard et al. 2017; Margutti et al. 2017; Troja et al. 2017; D'Avanzo et al. 2018), radio (Hallinan et al. 2017) and optical bands (Lyman et al. 2018), and an optical-IR thermal transient source (Evans et al. 2017; Valenti et al. 2017; Coulter et al. 2018). The latter transient allowed for the localization of the event with sub-arcsecond precision in the S0-type galaxy NGC 4993 at a distance of $40.7 \pm$ 3.3 Mpc (Palmese et al. 2017; Cantiello et al. 2018). This thermal transient showed evidence of heating as a result of $r$-process nucleosynthesis (Tanvir et al. 2017; Cowperthwaite et al. 2017; Pian et al. 2017; Smartt et al. 2017), classifying it as a "kilonova" $(\mathrm{KN})$, the first to feature such detailed observations.

According to estimates on joint GW-GRB events by Beniamini et al. (2019), such a combined detection of GWGRB-AG-KN should remain rare. Indeed, GRB170817A would not have been detected at a distance larger than $50 \mathrm{Mpc}$ (Goldstein et al. 2017) or from a viewing angle larger than $\sim 25^{\circ}$.

In the context of the present $\mathrm{O} 3$ and future observing runs of the LIGO-Virgo Collaboration (LVC), we would like to know what to expect regarding the afterglows of future binary neutron star (BNS) mergers: the rates of GW and joint GW-AG events; the distributions of event observables, such as distance $D$, viewing angle $\theta_{\mathrm{v}}$, radio afterglow time of peak $t_{\mathrm{p}}$ and peak flux $F_{\mathrm{p}}$, and remnant proper motion $\mu$; and the sensitivity of these to the population's characteristics and to the multi-messenger detector configuration.

In this paper, we approach these questions from the angle of a population study. More precisely, we start from the basis of prior knowledge on binary neutron star mergers from short GRB science and observations of GW170817 and its counterparts. This allows us to prescribe some intrinsic variability in mergers of the population, such as their kinetic energy, external medium density, shock microphysical conditions, etc. We then calculated the radio afterglows arising from this population of mergers in the hypothesis of a jet-dominated afterglow emission. Finally, we applied the thresholds of current and future GW and radio detectors to determine the events within the population which would be detectable in both the GW and radio domains. By describing and studying this jointly-detectable population, we make predictions for observing runs in the future.

Saleem et al. (2018a,b) consider such a population model for short GRB afterglows, with detailed numerical models for the multi-band afterglow and GW signals, and based on uninformative (e.g., uniform or log-uniform) event parameter distributions. Moreover, the discussion therein is centered around the parameter-space constraints that can be derived from detections or non-detections of the afterglow in various electromagnetic bands, and on the conditions necessary to observe afterglows from off-axis lines of sight. Here, we discuss, rather, the effect of the population parameters and detector configurations on the expected population of afterglows.

More recently, Gottlieb et al. (2019) also follows this approach. Once again, our study differs in that it accounts for 
some prior knowledge of short GRBs (such as their luminosity function) and observations from GW170817 to sharpen our predictions for future events. This allows for a detailed study of the impact of the population parameters on these predicted observations.

Our study relies on the following assumptions.

(i) In most cases the merger produces a successful central jet. This is clearly a strong assumption that will have to be validated by future observations. Recent studies based on the population of short GRBs (Beniamini et al. 2019) and the dynamics of jets interacting with merger ejecta (Duffell et al. 2018) suggest that successful jets could be common for these phenomena.

(ii) Around its peak, the afterglow is dominated by the contribution of the jet's core. In this case, the afterglow peak flux depends on the jet's core parameters (core isotropic kinetic energy $E_{\mathrm{iso}, \mathrm{c}}$ and opening angle $\theta_{\mathrm{j}}$, shock microphysics parameters $\epsilon_{\mathrm{e}}, \epsilon_{\mathrm{B}}$, and $p$ ), on its environment (external medium density $n$ ), and on its viewing conditions (the distance and viewing angle). The population's distributions of most of these quantities remain quite uncertain. Our knowledge of these relies on the afterglow fitting of a limited sample of short GRB afterglows with known distance (e.g., Berger 2014; Fong et al. 2015), on the expectation that short GRBs generally occur in a low-density environment, and on the results obtained from the (up to now) single multi-messenger event GRB170817A (e.g., van Eerten et al. 2019).

This publication is organized as follows. In Sect. 2 we describe our methods to calculate the afterglow emission and detail the input parameters of our population model. In Sect. 3 we provide the criteria for $\mathrm{GW}$ and radio afterglow detection that we applied to deduce the jointly-detectable population. In Sect. 4 we report general results on this population, describe its observable characteristics, and study their sensitivity to the detector configurations and to the population model parameters. In Sect. 5, we discuss our results in the context of future multi-messenger campaigns and how they may help to interpret observations therein.

\section{A population model for binary neutron star merger afterglows}

\subsection{Radio afterglows from structured jets}

The multi-wavelength afterglow of GW170817 is associated with the deceleration of a structured relativistic jet emitted by the central source formed in the merger, and more precisely, to the synchrotron emission of electrons accelerated by the forward shock propagating in the external medium. It was observed for more than 300 days. Its time evolution is similar at all wavelengths, indicating that the emission was produced in the same spectral regime of the slow-cooling synchrotron process, that is, $v_{\mathrm{m}}<v<v_{\mathrm{c}}$, and that the non-thermal distribution of shock-accelerated electrons assumed a non-evolving slope $p \sim 2.2$ (Nakar et al. 2018; Mooley et al. 2018a; van Eerten et al. 2019). The observed slow rise of the afterglow is clear evidence of the lateral structure of the outflow, which was observed off-axis with a viewing angle of $\theta_{\mathrm{v}} \sim 15-25^{\circ}$. Initially, the observed flux is dominated by the beamed emission from mildly-relativistic, mildly-energetic material coming towards the observer (Mooley et al. 2018b). As the jet decelerates, relativistic beaming becomes less efficient and regions closer to the jet axis start to contribute to the flux. The peak of the afterglow is reached when the jet's core, which is open to a few degrees at the angle $\theta_{\mathrm{j}}$, is revealed. This occurs when the jet's core's Lorentz factor has decreased to $\Gamma \sim 1 / \theta_{\mathrm{v}}$.

An alternative possibility is a quasi-spherical, mildly relativistic ejecta with a steep radial structure (Kasliwal et al. 2017; Gottlieb et al. 2018; Hotokezaka et al. 2018; Mooley et al. 2018b; Gill \& Granot 2018). This is now strongly disfavored by radio very long baseline interferometry (VLBI) observations, which demonstrate an apparent superluminal motion of the remnant with $\beta_{\text {app }} \sim 4$ (Mooley et al. 2018c) and constrain the angular size of the radio source to below 2 mas (Ghirlanda et al. 2019), thus confirming the emergence of a relativistic core jet at the peak of the afterglow.

The lateral structure of the jet is constrained by observations: the best fits are obtained for a sharp decay of the kinetic energy and Lorentz factor, with the angle $\theta$ from the jet axis (Lamb \& Kobayashi 2017; Lazzati et al. 2017; Troja et al. 2018; Resmi et al. 2018; Margutti et al. 2018). Typically, for a top-hat core jet with an opening angle $\theta_{\mathrm{j}}$, an isotropic equivalent kinetic energy $E_{\mathrm{iso}, \mathrm{c}}=4 \pi \epsilon_{\mathrm{c}}$ and Lorentz factor $\Gamma_{\mathrm{c}}$, surrounded by a power-law-structured sheath with the following kinetic energy per solid angle $\epsilon(\theta)$ and Lorentz factor $\Gamma_{0}(\theta)$,

$\epsilon(\theta)=\epsilon_{\mathrm{c}} \begin{cases}1 & \text { if } \theta \leq \theta_{\mathrm{j}} \\ \left(\theta / \theta_{\mathrm{j}}\right)^{-a} & \text { if } \theta \geq \theta_{\mathrm{j}}\end{cases}$

and

$\Gamma_{0}(\theta)=1+\left(\Gamma_{\mathrm{c}}-1\right)\left\{\begin{array}{ll}1 & \text { if } \theta \leq \theta_{\mathrm{j}} \\ \left(\theta / \theta_{\mathrm{j}}\right)^{-b} & \text { if } \theta \geq \theta_{\mathrm{j}}\end{array}\right.$,

observations require steep slopes of $a, b \gtrsim 2$ (Gill \& Granot 2018; Ghirlanda et al. 2019).

An example of the radio light curves obtained for such a structured jet is plotted in Fig. 1 (left) using parameters typical of the various fits to the data that have been published (see e.g., Gill \& Granot 2018; van Eerten et al. 2019; Ghirlanda et al. 2019). To obtain this light curve, the dynamics of the material at different latitudes are computed independently. At a latitude of $\theta$, the deceleration radius is $R_{\operatorname{dec}}(\theta)=\left(\frac{3 \epsilon(\theta)}{\Gamma_{0}^{2}(\theta) n m_{\mathrm{p}} c^{2}}\right)^{1 / 3}$, which is constant at the core and slowly increases with $\theta$ outside the core $\left(R_{\operatorname{dec}}(\theta) \propto \theta^{(2 b-a) / 3}\right)$. For the values of $a$ and $b$ chosen in Fig. 1, $\frac{2 b-a}{3}=0.17$.

Subsequently, the synchrotron emission of shock-accelerated electrons in the shock comoving frame is assumed to follow the standard synchrotron slow-cooling spectrum (Sari et al. 1998) including self-absorption. Finally, the observed light curve is computed by summing the contributions of all latitudes on equalarrival time surfaces, taking relativistic beaming and Doppler boosting into account.

The separate contributions of the core jet and the sheath are plotted in Fig. 1 (left) and the emergence of the core at the peak is clearly visible. The evolution of the peak flux of the same structured jet is plotted in Fig. 1 (right) as a function of the viewing angle, as well as the ratio of the peak flux to the peak flux from the core jet only. Interestingly, this ratio is almost constant $(\sim 1.5)$, except for the on-axis cases $\left(\theta_{\mathrm{v}} \leq \theta_{\mathrm{j}}\right)$ when the core is more dominant.

As long as the kinetic energy and the Lorentz factor decay steeply with $\theta$, the core jet is expected to dominate the flux at the peak whatever the viewing angle, even if the precise value of the total-to-core ratio at the peak may slightly vary depending on the details of the assumed lateral structure. Therefore, as 
R. Duque et al.: Predictions for radio afterglows of binary neutron star mergers
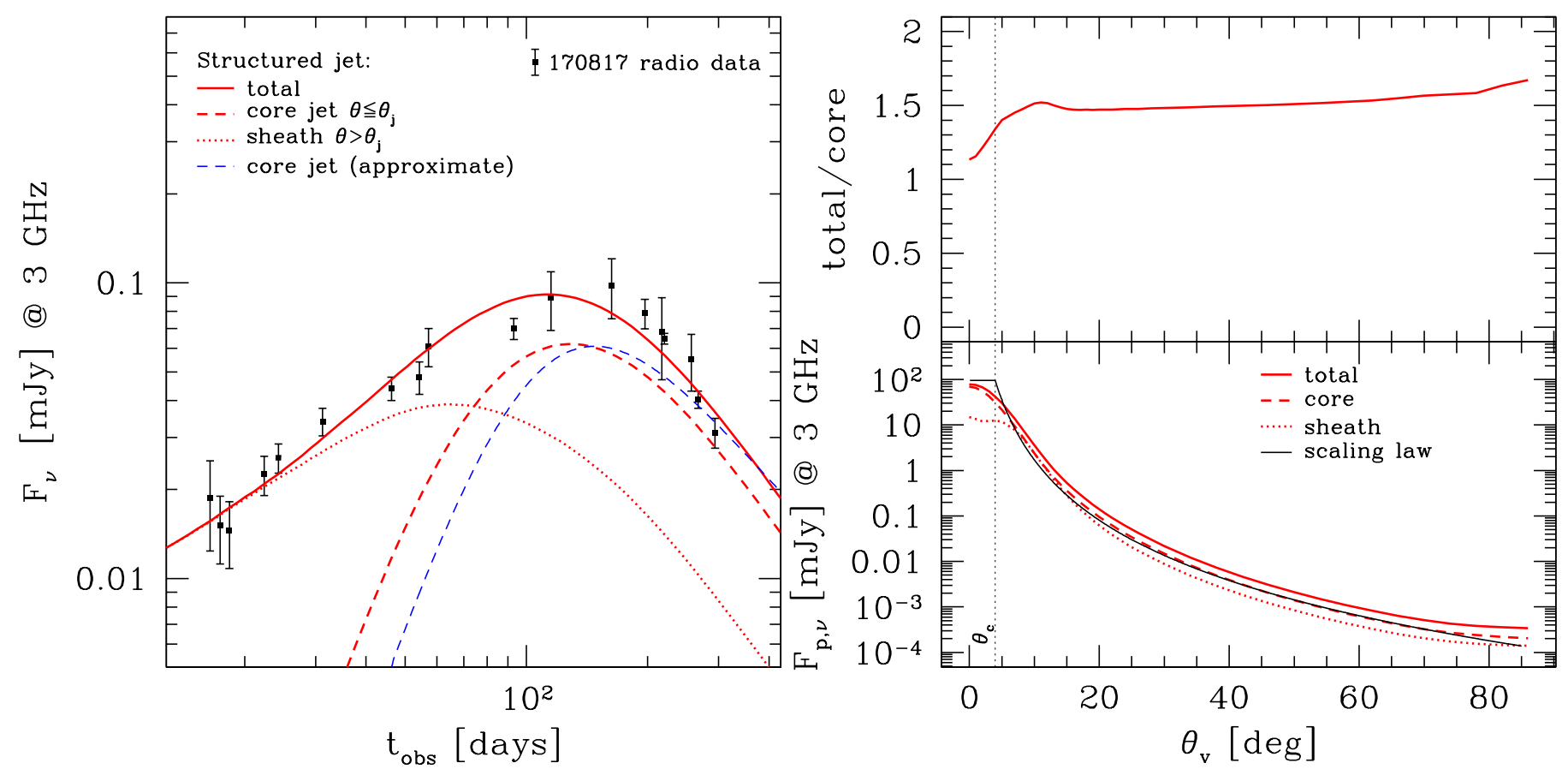

Fig. 1. Left: $3 \mathrm{GHz}$ light curve of afterglow from structured jet at distance $D=42 \mathrm{Mpc}$, with sharp power-law structure $(a=4.5, b=2.5$; see Eqs. (1)-(2)), viewing angle $\theta_{\mathrm{v}}=22^{\circ}$, external density $n=3 \times 10^{-3} \mathrm{~cm}^{-3}$, core jet with $\theta_{\mathrm{j}}=4^{\circ}, \Gamma_{\mathrm{c}}=100, E_{\mathrm{iso}, \mathrm{c}}=2 \times 10^{52}$ erg, and with microphysics parameters $p=2.2, \epsilon_{\mathrm{e}}=0.1$ and $\epsilon_{\mathrm{B}}=10^{-4}$. The radio observations of GW170817 are also plotted and are compiled from Hallinan et al. (2017), Alexander et al. (2018), Margutti et al. (2018), Mooley et al. (2018a,b), Dobie et al. (2018). The respective contributions of the core jet $\left(\theta \leq \theta_{\mathrm{j}}\right)$, the sheath and the total are plotted in dashed, dotted and solid red lines. The flux of the core jet computed with the simplified treatment described by Eq. (4) is also plotted in dashed blue line for comparison. Bottom right: peak flux of same structured jet as function of viewing angle $\theta_{\mathrm{v}}$ (solid red line), peak flux of light curve from core jet only (dashed red line) or from sheath only (dotted red line), and peak flux of core jet as computed using scaling law in Eq. (6) (thin black line). Upper right: ratio of peak flux from whole outflow (core jet+sheath) to that of core jet only.

our population model takes only the properties of the afterglow at the peak into account, that is, when it can be detected more easily, it is enough in the following to compute only the contribution from the core jet, while keeping in mind that it may slightly underestimate the total peak flux by a factor of ${ }^{1} \sim 1.5$.

The contribution from a top-hat core jet can efficiently be computed using the approximation suggested by Granot et al. (2002) to avoid the full integration over equal-arrival time surfaces. More precisely, we computed the flux $F_{v}^{\text {iso }}\left(t_{\text {obs }}\right)$ of a spherical ejecta with initial Lorentz factor $\Gamma_{\mathrm{c}}$ and kinetic energy $E_{\mathrm{iso}, \mathrm{c}}$ as in Panaitescu \& Kumar (2000), and we corrected it by defining the on-axis afterglow flux including the jet break:

$F_{v}^{\mathrm{on}}\left(t_{\mathrm{obs}}\right)=F_{v}^{\mathrm{iso}}\left(t_{\mathrm{obs}}\right) \times \begin{cases}1 & \text { if } \Gamma \theta_{\mathrm{j}} \leq 1 \\ \left(\Gamma \theta_{\mathrm{j}}\right)^{-2} & \text { if } \Gamma \theta_{\mathrm{j}} \geq 1\end{cases}$

and by calculating the afterglow from any viewing angle as:

$F_{v}\left(t_{\mathrm{obs}}\right)= \begin{cases}F_{v}^{\mathrm{on}}\left(t_{\mathrm{obs}}\right) & \text { if } \theta_{\mathrm{v}}<\theta_{\mathrm{j}} \text { (on axis) } \\ a^{3} F_{v / a}^{\mathrm{on}}\left(b t_{\mathrm{obs}}\right) & \text { if } \theta_{\mathrm{v}}>\theta_{\mathrm{j}}(\text { off axis })\end{cases}$

with $a=\frac{1-\beta}{1-\beta \cos \left(\theta_{\mathrm{v}}-\theta_{\mathrm{j}}\right)}$ and $b=\frac{1-\frac{R}{c t}}{1-\frac{R}{c t} \cos \left(\theta_{\mathrm{v}}-\theta_{\mathrm{j}}\right)}$ where $t$ is the source frame time. The latter correction, corresponding to the ratio of on-axis and off-axis arrival times is not included in Granot et al. (2002) and is found to improve the quality of the approximation. The corresponding light curve is plotted in Fig. 1 (left, blue dashed line) and corresponds well with the exact calculation. 1 See Sect. 5 for a short discussion of the effect of this on the fraction
of detectable events.

\subsection{An analytical model for afterglow peak properties}

This afterglow calculation is standard for top-hat jets. Therefore analytical expressions for the afterglow properties at the peak are available. They depend slightly on the assumptions for a possible late jet lateral expansion. If lateral expansion is taken into account like in standard GRB afterglow theory, the peak flux of the radio light curve scales as (Nakar et al. 2002):

$F_{\mathrm{p}, v} \propto E_{\mathrm{iso}, \mathrm{c}} \theta_{\mathrm{j}}^{2} n^{\frac{p+1}{4}} \epsilon_{\mathrm{e}}^{p-1} \epsilon_{\mathrm{B}}^{\frac{p+1}{4}} v^{\frac{1-p}{2}} D^{-2} \max \left(\theta_{\mathrm{j}}, \theta_{\mathrm{v}}\right)^{-2 p}$,

as long as the spectral regime remains $v_{\mathrm{m}}<v<v_{\mathrm{c}}$. This leads to the following expression ${ }^{2}$ of the peak flux at $3 \mathrm{GHz}$ :

$$
\begin{aligned}
F_{\mathrm{p}, 3 \mathrm{GHz}}= & 8.6 E_{52} \theta_{\mathrm{j},-1}^{2} n_{-3}^{4 / 5} \epsilon_{\mathrm{e},-1}^{6 / 5} \epsilon_{\mathrm{B},-3}^{4 / 5} \\
& \times D_{100}^{-2} \max \left(\theta_{\mathrm{j},-1}, \theta_{\mathrm{v},-1}\right)^{-4.4} \mathrm{mJy},
\end{aligned}
$$

where $E_{52}=E_{\text {iso,c }} / 10^{52} \mathrm{erg}, \theta_{\mathrm{j},-1}=\theta_{\mathrm{j}} / 0.1 \mathrm{rad}, n_{-3}=n /\left(10^{-3}\right.$ $\left.\mathrm{cm}^{-3}\right), \epsilon_{\mathrm{e},-1}=\epsilon_{\mathrm{e}} / 10^{-1}, \epsilon_{\mathrm{B},-3}=\epsilon_{\mathrm{B}} / 10^{-3}, \theta_{\mathrm{v},-1}=\theta_{\mathrm{v}} / 0.1 \mathrm{rad}$ and $D_{100}=D /(100 \mathrm{Mpc})$, and where we assume $p=2.2$. This scaling law is plotted in Fig. 1 (right, solid black line) and corresponds well with the detailed calculation. The expression of the corresponding peak time is also given by Nakar et al. (2002), assuming lateral expansion of the jet. However, even if late observations of GRB170817A may give some evidence for the lateral expansion of the core jet (for example the temporal decay index

\footnotetext{
2 We don't take into account the effect of redshift, as the events at play here are within the GW horizon distance of first generation interferometers, i.e., with $z \leq 0.1$.
} 
being $\sim-p$, as found by Mooley et al. 2018a), it is not clear if this expansion should be as strong for a core jet embedded in a sheath as for a "naked" top-hat jet. Therefore, we also consider a limit case without lateral expansion. This only affects the peak flux slightly and Eq. (6) above remains a good approximation in both cases. On the other hand, it modifies the peak time, leading to:

$$
\begin{aligned}
t_{\mathrm{p}, \text { no ex }} & \sim t_{\mathrm{b}} \times\left(\frac{\theta_{\mathrm{v}}}{\theta_{\mathrm{j}}}\right)^{8 / 3} \\
& =137\left(\frac{E_{52}}{n_{-3}}\right)^{1 / 3} \theta_{v, 35}^{8 / 3} \text { days }
\end{aligned}
$$

and

$$
\begin{aligned}
t_{\mathrm{p}, \mathrm{ex}} & \sim t_{\mathrm{b}} \times\left(\frac{\theta_{\mathrm{v}}}{\theta_{\mathrm{j}}}\right)^{2} \\
& =60\left(\frac{E_{52}}{n_{-3}}\right)^{1 / 3} \theta_{j,-1}^{2 / 3} \theta_{v, 35}^{2} \text { days }
\end{aligned}
$$

where $t_{\mathrm{b}}$ is the standard jet break time,

$t_{\mathrm{b}}=4.9\left(E_{52} / n_{-3}\right)^{1 / 3} \theta_{j,-1}^{8 / 3}$ days.

The ratio of the peak times without and with lateral expansion is simply

$$
\frac{t_{\mathrm{p}, \text { without }}}{t_{\mathrm{p} \text {,with }}} \sim\left(\frac{\theta_{\mathrm{v}}}{\theta_{\mathrm{j}}}\right)^{2 / 3} \text {. }
$$

In the following, we use, unless otherwise noted, Eqs. (6)-(8) to estimate the peak flux and peak time of the radio afterglow from a binary neutron star merger.

\subsection{Physical parameters of the jet and their intrinsic distribution}

To generate a population of jet afterglows we had to fix the various parameters appearing in Eqs. (6)-(8). We first defined a set of "fiducial distributions" for these parameters. These are summarized in Table 1 and have been chosen as follows.

Core jet isotropic equivalent kinetic energy $E_{\text {iso,c }}$ : we adopted a broken power-law distribution, which we deduced directly from the gamma-ray luminosity function of cosmological short GRBs, assuming a standard rest frame duration $\langle\tau\rangle=0.2 \mathrm{~s}$ and a standard efficiency $f_{\gamma}=0.2$ (Beniamini et al. 2016) so that $E_{\text {iso,c }} \sim L_{\gamma}\langle\tau\rangle / f_{\gamma}$, leading to a density of probability

$$
\begin{aligned}
\phi\left(E_{\mathrm{iso}, \mathrm{c}}\right) & =\frac{1}{N} \frac{\mathrm{d} N}{\mathrm{~d} E_{\mathrm{iso}, \mathrm{c}}} \\
& \propto \begin{cases}E_{\mathrm{iso}, \mathrm{c}}^{-\alpha_{1}} & \text { for } E_{\mathrm{min}} \leq E_{\mathrm{iso}, \mathrm{c}} \leq E_{\mathrm{b}} \\
E_{\mathrm{iso}, \mathrm{c}}^{-\alpha_{2}} & \text { for } E_{\mathrm{b}} \leq E_{\mathrm{iso}, \mathrm{c}} \leq E_{\max }\end{cases}
\end{aligned}
$$

where $\phi$ is normalized to unity. We fixed $E_{\min }=10^{50} \mathrm{erg}$ and $E_{\max }=10^{53} \mathrm{erg}$. Luminosity functions for short GRBs were deduced based on observations from several teams (e.g., D'Avanzo et al. 2014; Wanderman \& Piran 2015; Ghirlanda et al. 2016). For our fiducial model, we adopted that of Ghirlanda et al. (2016) with $\alpha_{1}=0.53, \alpha_{2}=3.4$ and a break luminosity of $L_{\mathrm{b}}=2 \times 10^{52} \mathrm{erg} \mathrm{s}^{-1}$ leading to $E_{\mathrm{b}}=2 \times 10^{52} \mathrm{erg}$.

Jet opening angle $\theta_{\mathrm{j}}$ : for simplicity we assumed a single value $\theta_{\mathrm{j}}=0.1 \mathrm{rad}$, which appears to be representative of the typical value found by fitting the afterglow light curve of GW170817 (e.g., Gill \& Granot 2018; van Eerten et al. 2019), by the VLBI observations of the remnant (Mooley et al. 2018c) and also consistent with the results of prior short GRB afterglow fitting (Fong et al. 2015) and short GRB-BNS merger rate comparisons (Beniamini et al. 2019).

External density $n$ : most BNS mergers are expected to occur in low density environments due to a long merger time, as assessed by the significant offsets of short GRB sources from their host galaxies (e.g., Nakar 2007; Troja et al. 2008; Fong et al. 2010; Church et al. 2011; Berger 2014). In the case of GW170817, afterglow fitting typically leads to $n \sim 10^{-3} \mathrm{~cm}^{-3}$, which is in agreement with the estimation of the HI content of the host galaxy NGC 4996 (Hallinan et al. 2017). The external densities observed in short GRBs cover the interval $10^{-3}-1 \mathrm{~cm}^{-3}$ (Berger 2014), but this is probably biased towards high densities, which favor afterglow detection. In this paper we consider $n \sim 10^{-3} \mathrm{~cm}^{-3}$ as typical and set, therefore, a log-normal of mean $10^{-3} \mathrm{~cm}^{-3}$ with a standard deviation of 0.75 as the fiducial density distribution.

Microphysics parameters: we set the value of $\epsilon_{\mathrm{e}}$ at 0.1 . This is generally done in GRB afterglow modeling (Wijers \& Galama 1999; Panaitescu \& Kumar 2001; Santana et al. 2014; Beniamini \& van der Horst 2017; D’Avanzo et al. 2018). Also, this value has been adopted or found in the fitting of the afterglow of GW170817 (e.g., van Eerten et al. 2019; Margutti et al. 2018; Nakar et al. 2018; Xie et al. 2018), and it is consistent with particle shock-acceleration simulations (Sironi \& Spitkovsky 2011). Similarly, we set $p$ at 2.2 , which is also a common value used in GRB afterglow models and which is in agreement with shock-acceleration theory in the relativistic regime (e.g., Sironi et al. 2015). In the case of GW170817 this value can be directly measured from multi-wavelength observations (Mooley et al. 2018a,b; van Eerten et al. 2019). Finally, more diversity is generally expected for $\epsilon_{\mathrm{B}}$. We assumed a log-normal distribution similar to the one adopted for $n$ with the additional constraint that $\epsilon_{\mathrm{B}}$ is restricted to the interval $\left[10^{-4}, 10^{-2}\right]$.

In Sect. 4.2 we consider possible alternatives to these fiducial distributions. In particular, we discuss the impact of substantial uncertainties in the distribution of kinetic energy, $\phi\left(E_{\mathrm{iso}, \mathrm{c}}\right)$, either due to the difficulty in measuring the luminosity function of short GRBs, or in simplifying assumptions regarding the duration and the efficiency of the prompt GRB emission. We also explore different values of the jet opening angle $\theta_{\mathrm{j}}$. As for the external density, we examine the effect of a change in the mean circum-merger density of the population of mergers.

\section{From the intrinsic to the observed population: GW and radio joint detection}

\subsection{GW detection criterion}

The signal-to-noise ratio $(\mathrm{S} / \mathrm{N}$, denoted by $\rho)$ of a $\mathrm{GW}$ inspiral signal in a single LIGO-Virgo-type interferometer can be written as $\rho^{2}=\frac{\Theta^{2}}{D^{2}} \mathcal{M}^{5 / 3} S_{\text {I }}$ (Finn \& Chernoff 1993), where $D$ is the luminosity distance to the binary, $\mathcal{M}$ is the chirp mass of the binary, $S_{\text {I }}$ is a quantity depending only on the sensitivity profile of the interferometer, and $\Theta^{2}$ represents the dependence of the signal to the binary sky position $(\theta, \phi)$ and orientation $\left(\theta_{\mathrm{v}}, \xi\right)$ with respect to the plane of the interferometer. Again, $\theta_{\mathrm{v}}$ is the angle of the line of sight to the binary polar direction, which we also assumed is the jet-axis direction. The function $\Theta^{2}$ admits a global maximum of $\Theta_{M}^{2}=16$ corresponding to an optimally positioned and oriented source (binary at the zenith and polar axis orthogonal to the instrument's arms). This optimal binary can be detected out 
Table 1. Fiducial population model parameter distributions.

\begin{tabular}{|c|c|c|}
\hline Parameter & Symbol & Probability distribution function \\
\hline Core jet isotropic equivalent kinetic energy & $E_{\mathrm{iso}, \mathrm{c}}$ & $\begin{array}{l}\text { Broken power law (Eq. (11)), } \\
\text { with } \alpha_{1}=0.53, \alpha_{2}=3.4 \\
\text { and } E_{\min }, E_{\mathrm{b}}, E_{\max }=10^{50}, 2 \times 10^{52}, 10^{53} \mathrm{erg}\end{array}$ \\
\hline Jet half-opening angle & $\theta_{\mathrm{j}}$ & $0.1 \mathrm{rad} \sim 5.7^{\circ}$ \\
\hline External medium density & $n$ & $\begin{array}{l}\text { Log-normal distribution, with central value } n_{0}=10^{-3} \mathrm{~cm}^{-3} \\
\text { and standard deviation } \sigma_{\mathrm{n}}=0.75\end{array}$ \\
\hline Electron redistribution parameter & $\epsilon_{\mathrm{e}}$ & Fixed at 0.1 \\
\hline Magnetic field redistribution parameter & $\epsilon_{\mathrm{B}}$ & Same log-normal as $n$, restricted to $\left[10^{-4}, 10^{-2}\right]$ \\
\hline Electron population spectral index & $p$ & Fixed at 2.2 \\
\hline
\end{tabular}

to a distance known as the horizon $H$ of the instrument, which depends on the $\mathrm{S} / \mathrm{N}$ threshold for detection, taken to be $\rho_{0}=8$ for the LVC network. We may thus rewrite the $\mathrm{S} / \mathrm{N}$ in the following manner:

$\rho^{2}=\rho_{0}^{2} \frac{\Theta^{2}}{\Theta_{M}^{2}} \frac{H^{2}}{D^{2}}$.

In a full population study, one would have to draw all four angles and evaluate this criterion in every case. We chose to reduce the number of parameters to two: $D$ and $\theta_{\mathrm{v}}$, as these are the ones relevant to the afterglow. Thus, we were compelled to review this criterion by averaging $\Theta^{2}$ on the sky-position $(\theta, \phi)$ and polarization angle $\xi$. This is done readily from the expression given in Finn \& Chernoff (1993; Eq. (3.31)) and it is found analytically that:

$\left\langle\Theta^{2}\right\rangle_{\xi, \theta, \phi}=\frac{1}{4 \pi 2 \pi} \int \mathrm{d} \xi \mathrm{d} \Omega \Theta^{2}=\frac{4}{5}\left(1+6 \cos ^{2} \theta_{\mathrm{v}}+\cos ^{4} \theta_{\mathrm{v}}\right)$.

Hence, we chose to use the following criterion to determine those binaries of inclination $\theta_{\mathrm{v}}$ and distance $D$ which are detectable in $\mathrm{GW}$ on average in the sky:

$\left\langle\rho^{2}\right\rangle_{\xi, \theta, \phi}>\rho_{0}^{2}$,

which is:

$\sqrt{\frac{1+6 \cos ^{2} \theta_{\mathrm{v}}+\cos ^{4} \theta_{\mathrm{v}}}{8}}>\frac{D}{\bar{H}}$

where we have denoted $\bar{H}=\sqrt{\frac{2}{5}} H \sim H / 1.58$ for the skyposition-averaged horizon.

Averaging $\Theta^{2}$ on inclination angle $\theta_{\mathrm{v}}$ and imposing an $\mathrm{S} / \mathrm{N}$ threshold as in Eq. (14) would lead to the simple detection criterion of $D<R$, where $R$ is the range of the instrument, that is, the maximum distance at which a binary can be detected on average in sky-position and in orientation. The range is linked to the horizon by $H=2.26 R$.

The criterion of Eq. (15) is valid for a detection using a single instrument. Instead, GW detection by the interferometer network is based on multi-instrument analysis, and is, thus, more complicated than the one described by our criterion. Furthermore, as was illustrated in the case of GW170817, true joint GW-AG detection requires pinpointing the source through the detection of the kilonova counterpart ${ }^{3}$, which in turn involves

\footnotetext{
3 This was not achieved in the recent merger candidates S190425z and S190426c (Ligo Scientific Collaboration \& VIRGO Collaboration 2019a,b), see Sect. 5 for more discussion on this.
}

Table 2. Horizons used for GW detection-localization criterion in our study.

\begin{tabular}{lll}
\hline \hline LVC run & $H(\mathrm{Mpc})$ & $\bar{H}=H \times \sqrt{2 / 5}(\mathrm{Mpc})$ \\
\hline O2 & 136 & 85.8 \\
O3 & 226 & 143 \\
Design & 429 & 272 \\
\hline
\end{tabular}

Notes. They are deduced from the published BNS ranges of the instruments (Abbott et al. 2018) by $H=2.26 R$ and considering the value for LIGO-Hanford (see text for details of this choice).

a small enough localization map from the GW data, and our criterion should incorporate this. We chose a simple localization criterion by supposing that a source is localized if it is detectable (once again on average) by the two most sensitive interferometers of the network. Thus our detection-localization criterion is that of Eq. (15), with the horizon of the second most sensitive instrument of the network, that is, the LIGOHanford instrument. Table 2 reports the corresponding values taken for our study for the LVC O2, O3 runs, and for the design interferometers.

In this framework, it can be shown that assuming homogeneity of the sources within the horizon and isotropy of the binary polar direction, the mean viewing angle of GW-detected events is $\sim 38^{\circ}$ and the fraction of GW-detected events among all mergers is $\sim 29 \%$, both regardless of the horizon value. This latter fraction is, thus, an absolute maximum for the fraction of mergers to be jointly detected in both radio and GW channels.

\subsection{Radio detection criterion}

The detection criterion in the radio band is simply for the $3 \mathrm{GHz}$ peak flux, as determined by our models, to be larger than the sensitivity of the radio array available for follow-up at the time of consideration, which we denote by $s$. We note that this is a criterion aimed rather at detectability than detection. True detection only occurs if observations are pursued for long enough after the GW merger signal, as the flux rises to its maximum. We also note that events which are marginally detectable would only provide poor astrophysical output because the inference of jet parameters requires the fitting of an extended portion of the radio afterglow light curve.

We took three typical radio sensitivities reflecting the present and future capabilities of radio arrays: (i) the Karl Jansky Very Large Array (VLA) at $s=15 \mu \mathrm{Jy}$, (ii) the phase I of the Square Kilometer Array (SKA1, Braun 2014), at $s=3 \mu \mathrm{Jy}$, and (iii) 

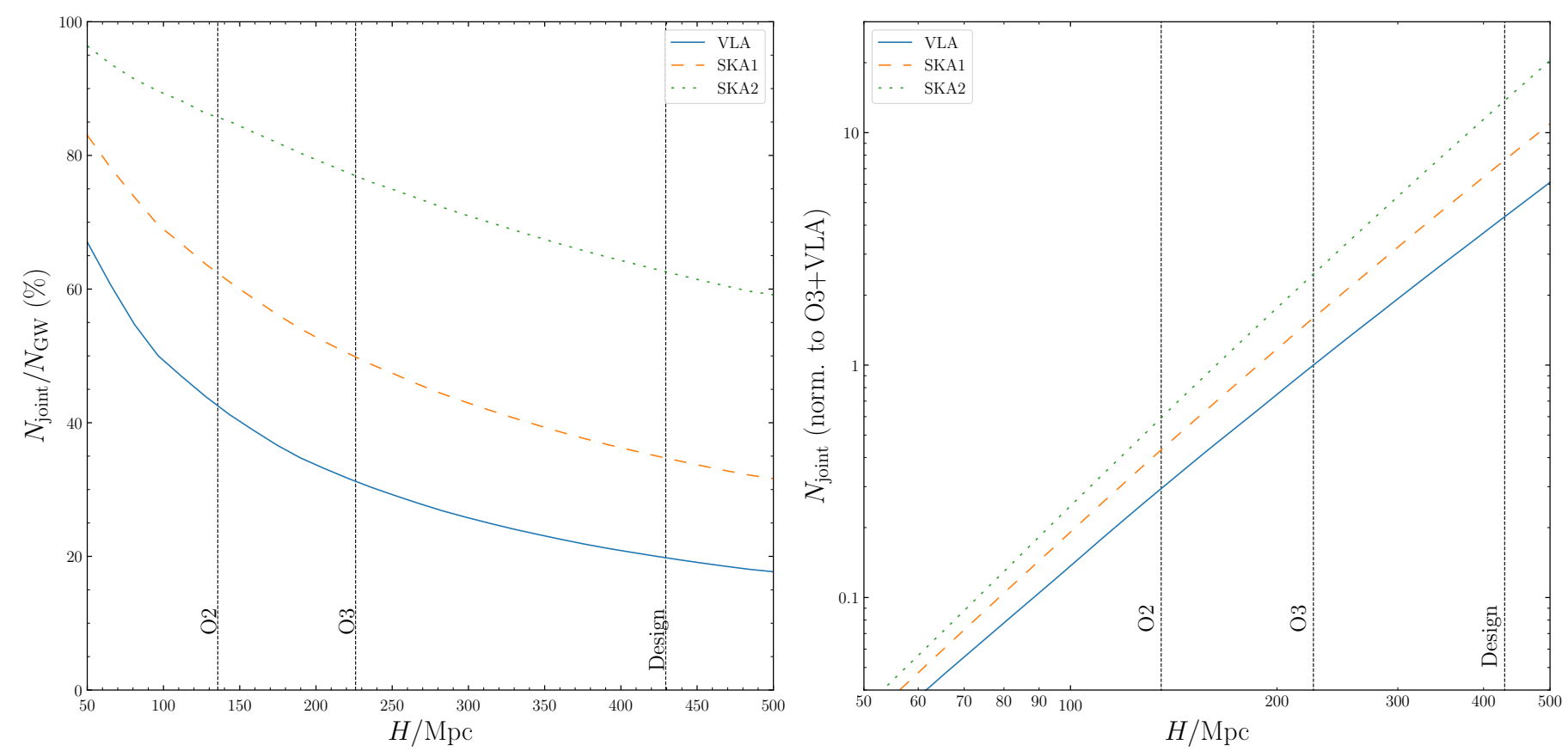

Fig. 2. Left: detectable fraction of radio afterglows among gravitational wave events as function of the horizon distance $H=1.58 \bar{H}$. Right: expected number of joint detections normalized to case of O3-VLA configuration. In both panels the full (resp. dashed, resp. dotted) lines correspond to the VLA (resp. SKA1, resp. SKA2/ngVLA) being the limiting radio facility.

phase II of the SKA (SKA2) and the Next Generation VLA (ngVLA, Selina et al. 2018), both at $s=0.3 \mu \mathrm{Jy}$. The combination of this radio detection criterion with that of the GW domain (Eq. (15)) constituted our criterion for the joint detection of mergers.

In the sequel, we referred to a jointly GW and radio detectable population by using the names of the corresponding $\mathrm{LVC}$ run and limiting radio facility, as in "the joint population of the $\mathrm{O} 3$ and VLA configuration". The corresponding GW interferometer horizons and radio sensitivities can be found respectively in Table 2 and in the paragraph above.

\section{Results: detection rates and properties of the detected population}

\subsection{Fiducial model}

In this section, we describe the population of events detected jointly in the GW and radio domains. For this purpose, we used a Monte Carlo approach in which we simulated $N>10^{6}$ binary systems within the sky-averaged horizon $\bar{H}$ using the parameter distributions of the fiducial model given in Sect. 2.3. By applying the detection criteria described in Sect. 3, we obtained $N_{\mathrm{GW}}$ systems detected by the GW interferometer network and $N_{\text {joint }}$ systems which are also detectable by radio telescopes by their afterglows. Then we studied the properties of the sample of joint GW and radio detectable events.

\subsubsection{Rate of joint GW and radio detectable events}

Starting from the fraction $f_{\mathrm{GW}}=N_{\mathrm{GW}} / N \sim 29 \%$ of binary systems detected by the LVC (Sect. 3.1), we then estimated which fraction $N_{\text {joint }} / N_{\mathrm{GW}}$ of those events would also produce a detectable radio afterglow.

This fraction is shown in Fig. 2 (left) as a function of the GW horizon and for the VLA, SKA1 and SKA2/ngVLA limiting sensitivities. In the design configuration of the LVC network, $75 \%$ (resp. 2 times) more events are detectable by SKA1 (resp. SKA2/ngVLA) than by the VLA.

The number of joint event detections normalized to the $\mathrm{O} 3$ VLA configuration is represented in Fig. 2 (right). The number of joint detections behaves approximately as $H^{\alpha}$, with $\alpha<3$ because of the reduction in radio detection efficiency when the distance is increased (Fig. 2, left). We find $\alpha \simeq 2.4$ (resp. 2.6, resp. 2.8) for the VLA (resp. SKA1, resp. SKA2/ngVLA).

As illustrated in Fig. 2 (left), we find that the fraction of detected events decreases from $43 \%(\mathrm{O} 2)$ to $31 \%(\mathrm{O} 3)$ with the VLA limiting sensitivity. However the absolute number of detections increases (Fig. 2, right). With the design-SKA1 configuration, a fraction of $35 \%$ of $\mathrm{GW}$ events can lead to a radio detection. With regard to the much higher sensitivities that may be reached after 2030, more than a half of GW events may become detectable in the radio band. Indeed we found a percentage of $63 \%$ of detectable joint events in the designSKA2/ngVLA configuration.

For the purposes of a comprehensive survey, the number of GW and jointly detected events per continuous year of GW network operation for future detector configurations can be found in Table 3, where we have used the horizons of Table 2 and our fiducial model.

\subsubsection{Distance and viewing angle}

The distributions of distances are shown in Fig. 3 for the O2-VLA and O3-VLA configurations, as well as for the entire GW population. In this figure, the distributions are not normalized to unity so as to show the decrease in the fraction of joint event as the horizon increases. It can be observed that as a result of the GW and radio detection thresholds, sources are progressively lost when the distance increases so that this distribution exhibits a near linear increase (as opposed to the $\mathrm{d} N / \mathrm{d} D \propto D^{2}$ of the intrinsic homogeneous population). Up to $D=\bar{H} / \sqrt{8} \simeq 0.35 \bar{H}$, all events are 
Table 3. Number of detectable events per continuous year of GW network operation.

\begin{tabular}{|c|c|c|c|c|c|}
\hline \multirow[t]{2}{*}{ LVC run } & \multicolumn{2}{|c|}{ Radio configuration } & \multirow{2}{*}{$\begin{array}{l}\text { GW events } \\
N_{\mathrm{GW}}\end{array}$} & \multirow{2}{*}{$\begin{array}{l}\text { Joint events } \\
N_{\text {joint }}\end{array}$} & \multirow{2}{*}{$\begin{array}{l}\text { Fraction of detectable events } \\
\text { (assuming fiducial model) }\end{array}$} \\
\hline & Instrument & $s(\mu \mathrm{Jy})$ & & & \\
\hline $\mathrm{O} 3$ & VLA & 15 & $9_{-7}^{+19}$ & $3_{-2}^{+6}$ & $31.4 \%$ \\
\hline Design & VLA & 15 & $21_{-16}^{+144}$ & $4_{-4}^{+10}$ & $19.8 \%$ \\
\hline Design & SKA1 & 3 & $21_{-16}^{+49}$ & $7_{-7}^{+18}$ & $34.7 \%$ \\
\hline Design & SKA2/ngVLA & 0.3 & $21_{-16}^{+160}$ & $13_{-13}^{+33}$ & $62.5 \%$ \\
\hline
\end{tabular}

Notes. The expected rate of total GW events is taken from Abbott et al. (2018) and values for joint events are derived using our fiducial population model. The uncertainties here are due to the uncertainty regarding the merger rate in the local Universe derived from GW observations of the LVC $\mathrm{O} 1$ and $\mathrm{O} 2$ runs. The additional uncertainties related to the population model are discussed in Sect. 4.2.
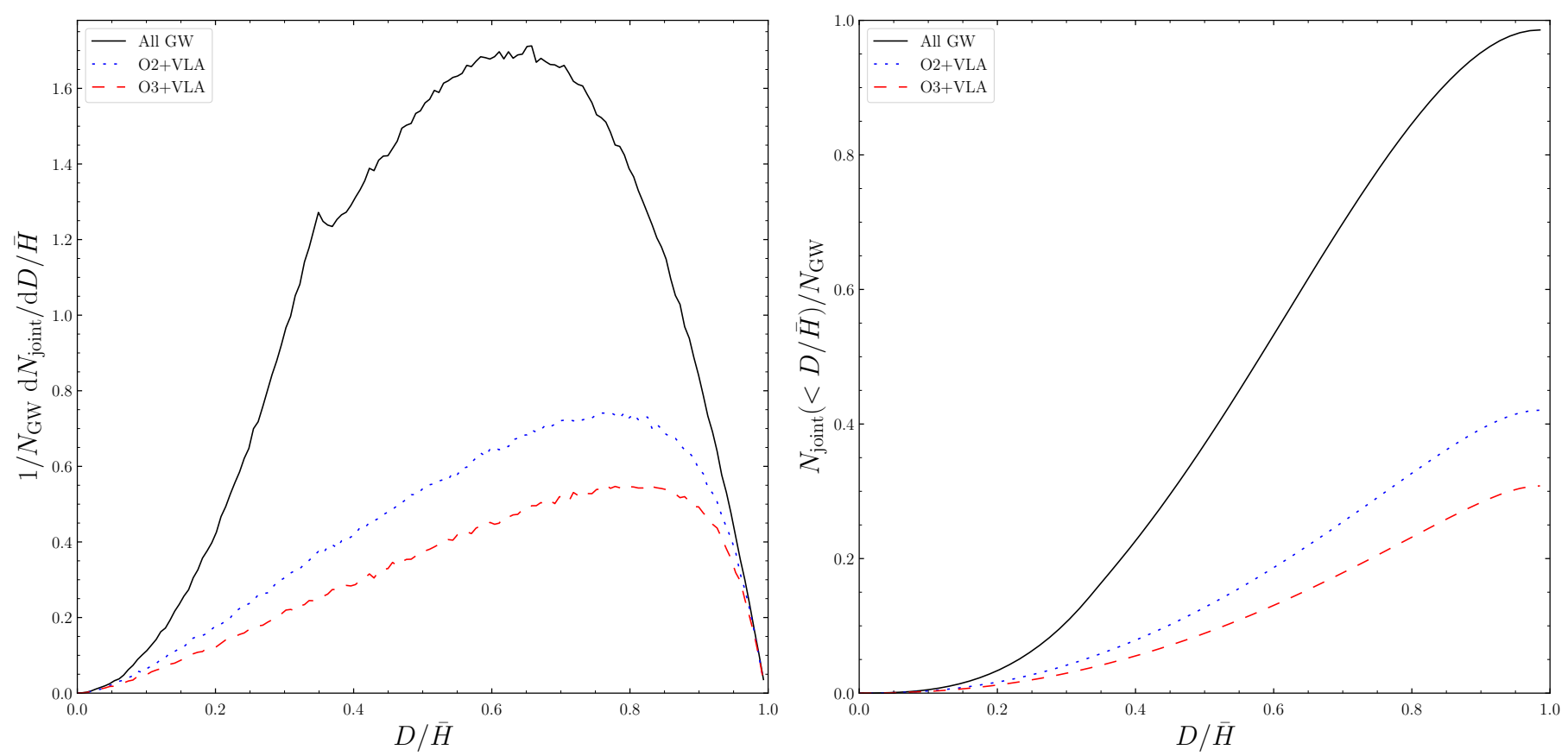

Fig. 3. Left: differential distribution of distances (normalized to the sky-position-averaged horizon) of events in total GW population (full line), and detectable jointly in O2-VLA (dotted line) and O3-VLA (dashed line) configurations. Right: cumulative distribution of same events. Distributions have been normalized to the fraction of jointly detected events among all GW-detected events, see text for details.

detected in $\mathrm{GW}$, regardless of their orientation ${ }^{4}$ and the only selection is due to the radio sensitivity. Near the horizon, the maximum viewing angle allowing GW-detection is $\theta_{\max } \propto \sqrt{1-D / \bar{H}}$, which produces a strict decrease of event density.

Figure 4 shows the distribution of the viewing angles. For the joint events, the peak of the distribution takes place at small viewing angles $\theta_{\mathrm{v}} \sim 15-20^{\circ}$ as a result of the rapid decline of the peak flux with angle $\left(F_{\mathrm{p}} \propto \theta_{\mathrm{v}}^{-2 p}\right)$, and about $39 \%$ (resp. $47 \%$ ) of the events would be seen with a viewing angle $\theta_{\mathrm{v}}<20^{\circ}$ for O2VLA (resp. O3-VLA), the angle at which the GW170817 event was likely to have been seen. As shown in Fig. 5 (left), the mean viewing angle of the jointly observed events strongly depends on the GW horizon and the radio sensitivity. It appears that even if the radio sensitivity is not substantially improved while the GW

\footnotetext{
4 Beyond this distance, events are detected in GW only if $\theta_{\mathrm{v}} \leq \theta_{\max }$ with $\cos \theta_{\max }=\sqrt{-3+\sqrt{8+8 D^{2} / \bar{H}^{2}}}$. The differential distribution of distances for the GW events thus transitions from $\propto D^{2}$ before $\bar{H} / \sqrt{8}$ to a non-quadratic form afterwards, producing the small peak seen in Fig. 3. This, of course, is only a consequence of our simplified GW skyaveraged detection criterion (Eq. (15)).
}

interferometers reach their design horizons, a significant number of events would still be observable off-axis. The increasing and decreasing phases of the afterglows of these events would allow for an improved study of the jet structure, which is better revealed by off-axis events. Figure 5 (right) shows the fraction of on-axis events $\left(\theta_{\mathrm{v}} \leq \theta_{\mathrm{j}}\right)$ within the joint detections. It increases from $4.0 \%$ (O2-VLA) to $5.3 \%$ (O3-VLA).

\subsubsection{Peak times, peak fluxes and synchrotron spectral regime}

The distributions of peak times and peak fluxes in the $\mathrm{O} 3$ VLA configuration are represented in Fig. 6. The fraction of events with a peak time of fewer than 150 days (as observed in GRB 170817A) is 55\% without jet expansion and $81 \%$ with lateral expansion. The distribution in peak flux is shown for all sources which are detected in gravitational waves within the sky-position-averaged horizon of $\mathrm{O} 2, \mathrm{O} 3$ and design instruments. It appears clear that for the present VLA sensitivity, most radio afterglows cannot be detected. This explains why improving the sensitivity of the future ngVLA would have such an impact on the joint detection rates, as shown in Fig. 2. 

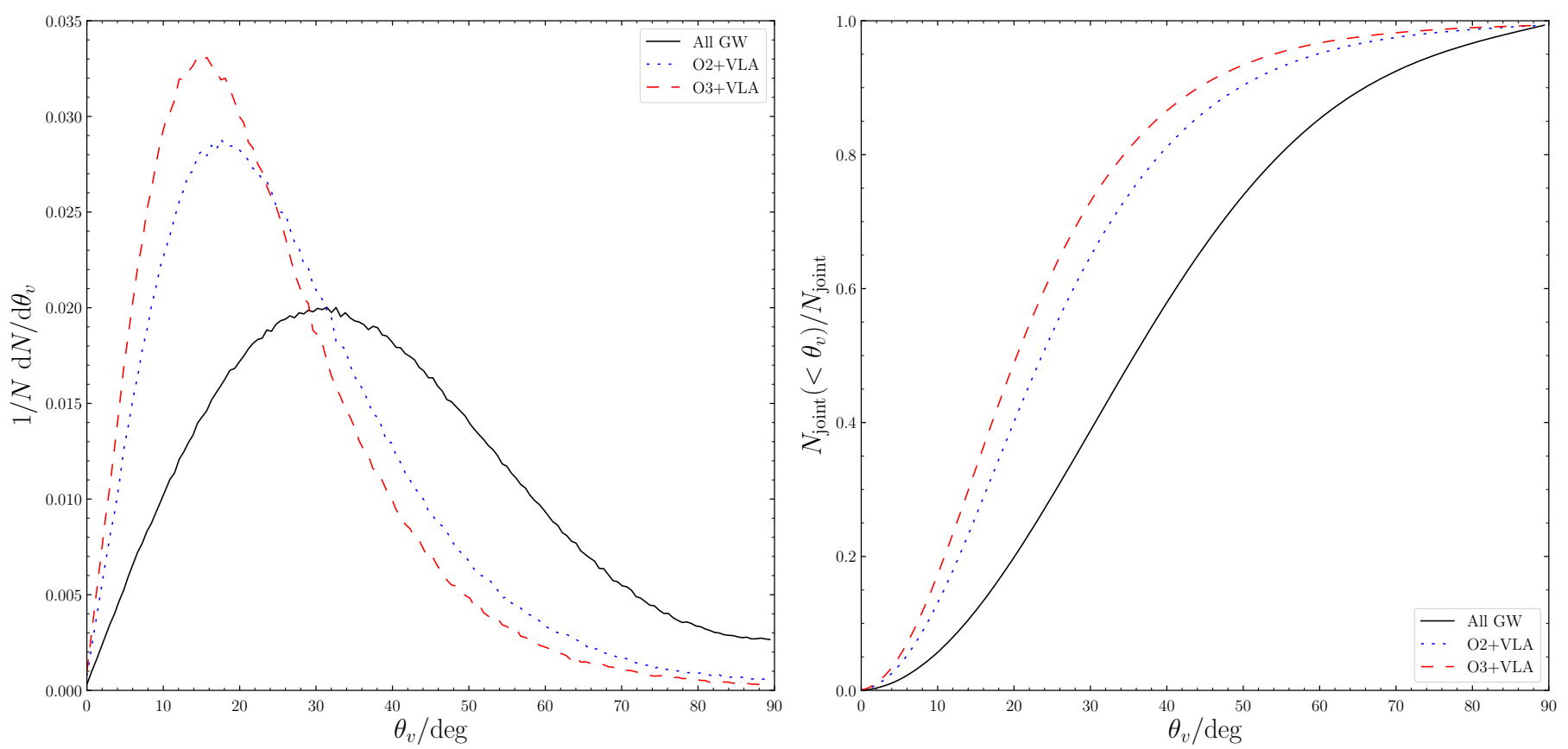

Fig. 4. Same as Fig. 3, for viewing angle. Distributions are normalized.
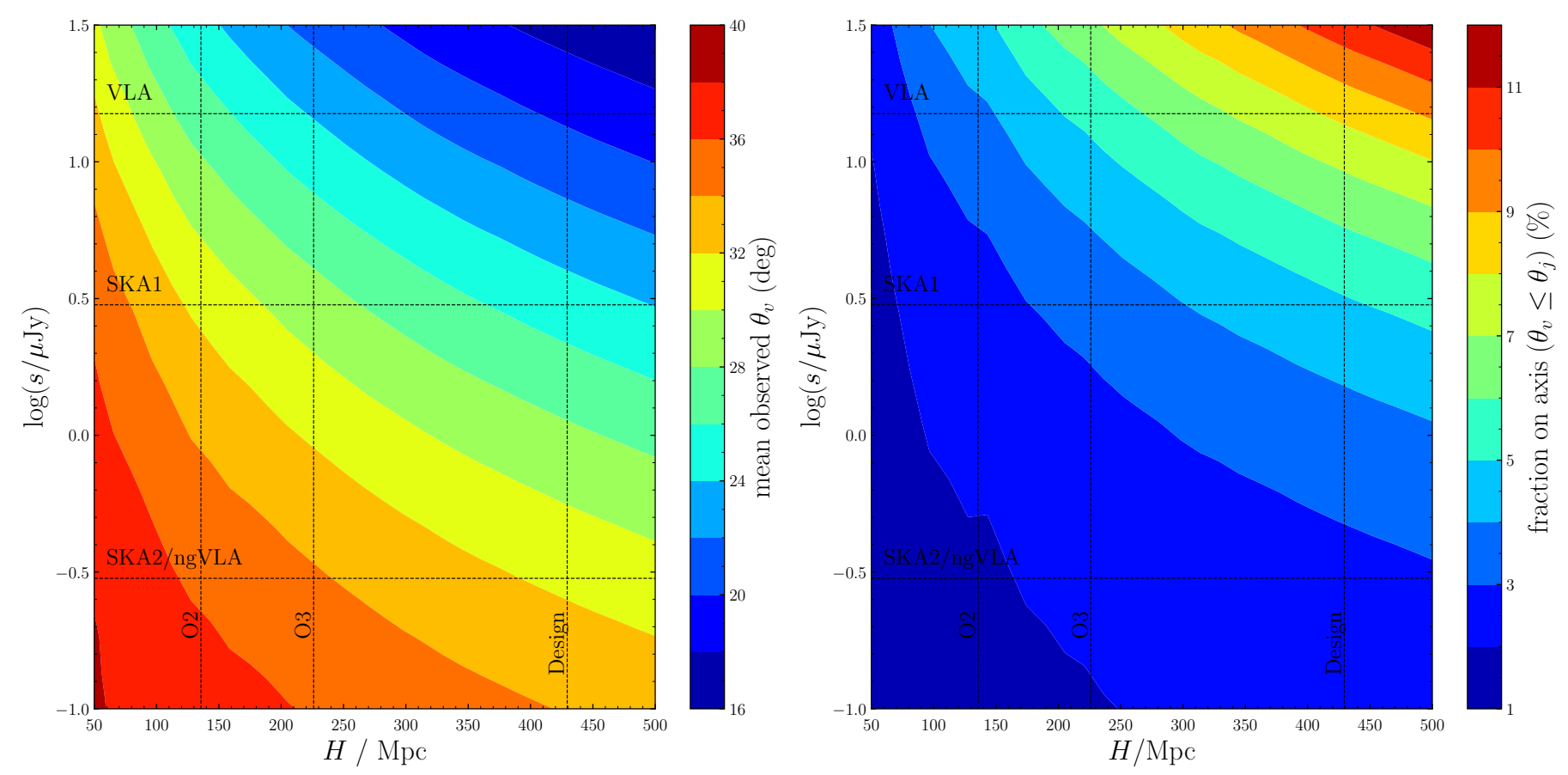

Fig. 5. Left: mean viewing angle of jointly detected events, as function of horizon and radio sensitivity $s$. Values of $H$ for $\mathrm{O} 2$, O3 and design instruments, as well as $s$ for VLA, SKA1 and SKA2/ngVLA, are indicated as dashed lines. Right: fraction of on-axis events (defined by $\theta_{\mathrm{v}} \leq \theta_{\mathrm{j}}$ ) among jointly detected events in same $H-s$ diagram.

The scaling law used to compute the radio emission at the peak assumes that the observing frequency remains in the same spectral regime of the slow-cooling synchrotron spectrum, that is, $v_{\mathrm{m}}<v<v_{\mathrm{c}}$, and above the absorption frequency. Using our more detailed calculation of the radio afterglow from the core jet (Eq. (4) and accompanying text), we can ascertain if this condition is fulfilled for the bulk of the population. However, for mergers in high density environments (larger than $10 \mathrm{~cm}^{-3}$ ), this condition is no longer met as the absorption frequency $v_{a}$ and the injection frequency $v_{i}$ may be larger than the radio frequency at early times.
For an event with $n=10 \mathrm{~cm}^{-3}$ and $\theta_{\mathrm{v}} \sim 40^{\circ}$ (the mean viewing angle of the GW-detected population), the radio frequency typically meets the injection break at around 30 days (resp. before 10 days). In this case, chromatic light curves are expected.

\subsubsection{Proper motion}

Another independent observable is the proper motion of the remnant, which can be readily accessible to VLBI measurements as illustrated in the case of GRB170817A (Mooley et al. 2018c; 

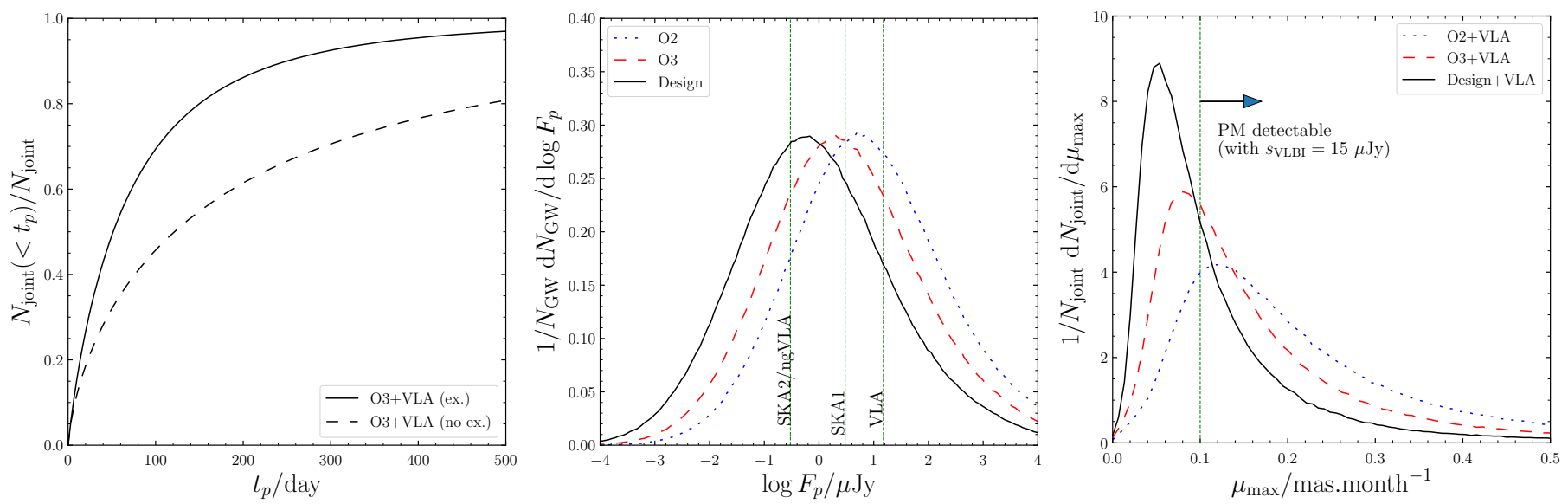

Fig. 6. Left: cumulative distributions of peak times of radio afterglows for jointly detected events in O3-VLA configuration, assuming jet with (solid line) or without (dashed line) lateral expansion. Middle: differential distribution of peak fluxes of GW-detected events assuming horizon for $\mathrm{O} 2$ (dotted line), $\mathrm{O} 3$ (dashed line) and design (full line) configurations of GW interferometers. The radio sensitivities for VLA, SKA1 and SKA2/ngVLA are indicated for comparison. Right: differential distribution of maximum proper motion for jointly detected events assuming O2VLA (dotted line), O3-VLA (dashed line), design-SKA1 (full line) configurations. The dotted vertical line shows the lower limit for detection of the proper motion.

Ghirlanda et al. 2019). However, a VLBI network can only measure the proper motion of a source if the total displacement of the source over the time it is detectable by the array is larger than the angular resolution of the network. For our purposes, we chose to simplify this criterion and adopt a dual condition on the flux $\left(F_{\mathrm{p}}>s_{\mathrm{VLBI}}\right)$, as well as on the instantaneous proper motion $\mu_{\max }$ (requiring that $\mu_{\max }>\mu_{\text {lim }}$ ) of the remnant at the peak of the afterglow. Here, we adopted a limiting proper motion of $\mu_{\mathrm{lim}}=3 \mu \mathrm{as} / \mathrm{day}$, in coherence with the uncertainties quoted in Mooley et al. (2018c), Ghirlanda et al. (2019).

An upper limit of the proper motion was obtained based on the assumption that the core jet contribution dominates at the peak of the radio light curve, and that the shocked region at the front of this core jet constitutes the visible remnant of the merger. In first approximation, afterglows from jets peak when the observer enters the focalization cone of the radiation, that is, $\Gamma \sim 1 / \theta_{\mathrm{v}}$. For an off-axis observer, we estimated, therefore, the maximum proper motion to be:

$\mu_{\max }=\frac{c \beta_{\mathrm{app}}}{D}=\frac{c}{D} f\left(\theta_{\mathrm{v}}\right)$

with

$$
f\left(\theta_{\mathrm{v}}\right)=\frac{\sqrt{1-\theta_{\mathrm{v}}^{2}} \sin \theta_{\mathrm{v}}}{1-\sqrt{1-\theta_{\mathrm{v}}^{2}} \cos \theta_{\mathrm{v}}} .
$$

Thus, for a given value of the viewing angle, there is a maximum distance beyond which the measurement of the proper motion is not possible. It is given by

$D_{\max }\left(\theta_{\mathrm{v}}\right)=\frac{c}{\mu_{\mathrm{lim}}} f\left(\theta_{\mathrm{v}}\right) \sim 50 f\left(\theta_{\mathrm{v}}\right) \mathrm{Mpc}$.

The distribution of $\mu_{\max }$ is shown in Fig. 6 (right) for $\mathrm{O} 2$, O3 and design-level detectable GW events respecting the VLBI flux criterion. Here, we have take $s_{\mathrm{VLBI}}=15 \mu \mathrm{Jy}$, which represents a limiting $\mathrm{S} / \mathrm{N}$ of $\sim 3$ with the level of noise reported in Mooley et al. (2018c), Ghirlanda et al. (2019) for the e-MERLIN and High Sensitivity Array networks. Within the O2-VLA (resp. O3-VLA, resp. design-VLA) populations, the measurement of the proper motion is possible in only $79 \%$ (resp. 64\%, resp. 42\%) of the jointly-detectable events.

Finally, we represent in Fig. 7 various plots connecting two observable quantities: $\left(\theta_{\mathrm{v}}, D\right),\left(\theta_{\mathrm{v}}, F_{\mathrm{p}, 3 \mathrm{GHz}}\right),\left(\theta_{\mathrm{v}}, t_{\mathrm{p}}\right)$ and $\left(D, \mu_{\max }\right)$. The grey dots correspond to events detectable in GW and the red crosses correspond to those detectable in both $\mathrm{GW}$ and radio. In the $\left(\theta_{\mathrm{v}}, D\right)$ diagram, the limiting distance for the measurement of the proper motion (Eq. (18)) is shown. This figure illustrates the various observational biases discussed in this section, and especially the bias towards small viewing angles, mainly due to the limiting sensitivity of radio telescopes.

\subsubsection{Kinetic energy, external density and microphysics parameters}

The distributions of kinetic energy $E_{\text {iso,c }}$ and external density $n$, which are not directly observable but may result from fits of the afterglow, are shown in Fig. 8. In each case, the distributions of jointly detected events have been compared to total GW detections. As the GW selection is independent of the kinetic energy and density, these distributions are the intrinsic distributions of our population model (Table 1). As could be expected, the detection of mergers leading to a relativistic core jet with a large $E_{\mathrm{iso}, \mathrm{c}}$ is favored. In jointly detectable mergers, the energy distribution approximately remains a broken power-law, but the respective indices below and above the break decrease from 0.53 to 0.2 and 3.4 to 3.2 , respectively. After applying the joint detectability criterion, the external density distribution is simply shifted to higher densities, as the mean value is increased by a factor of $\sim 2.2$. The distribution of the microphysics parameter $\epsilon_{\mathrm{B}}$ is very similar to that of the density, because both parameters appear with the same power in the peak flux (Eq. (6)).

We find that the Lorentz factor of the jet at the time of peak flux has a median value of $\sim 4$. This is consistent with the approximate jet core de-beaming relation $\Gamma \sim\left(\theta_{\mathrm{v}}-\theta_{\mathrm{j}}\right)^{-1}$ for a jet with $\theta_{\mathrm{j}}=0.1 \mathrm{rad}$ seen with the mean viewing angle $\theta_{\mathrm{v}} \sim 24^{\circ}$ for the O3-VLA configuration. This justifies keeping microphysics parameters representative of shock-acceleration in the ultra-relativistic regime for the whole population. 

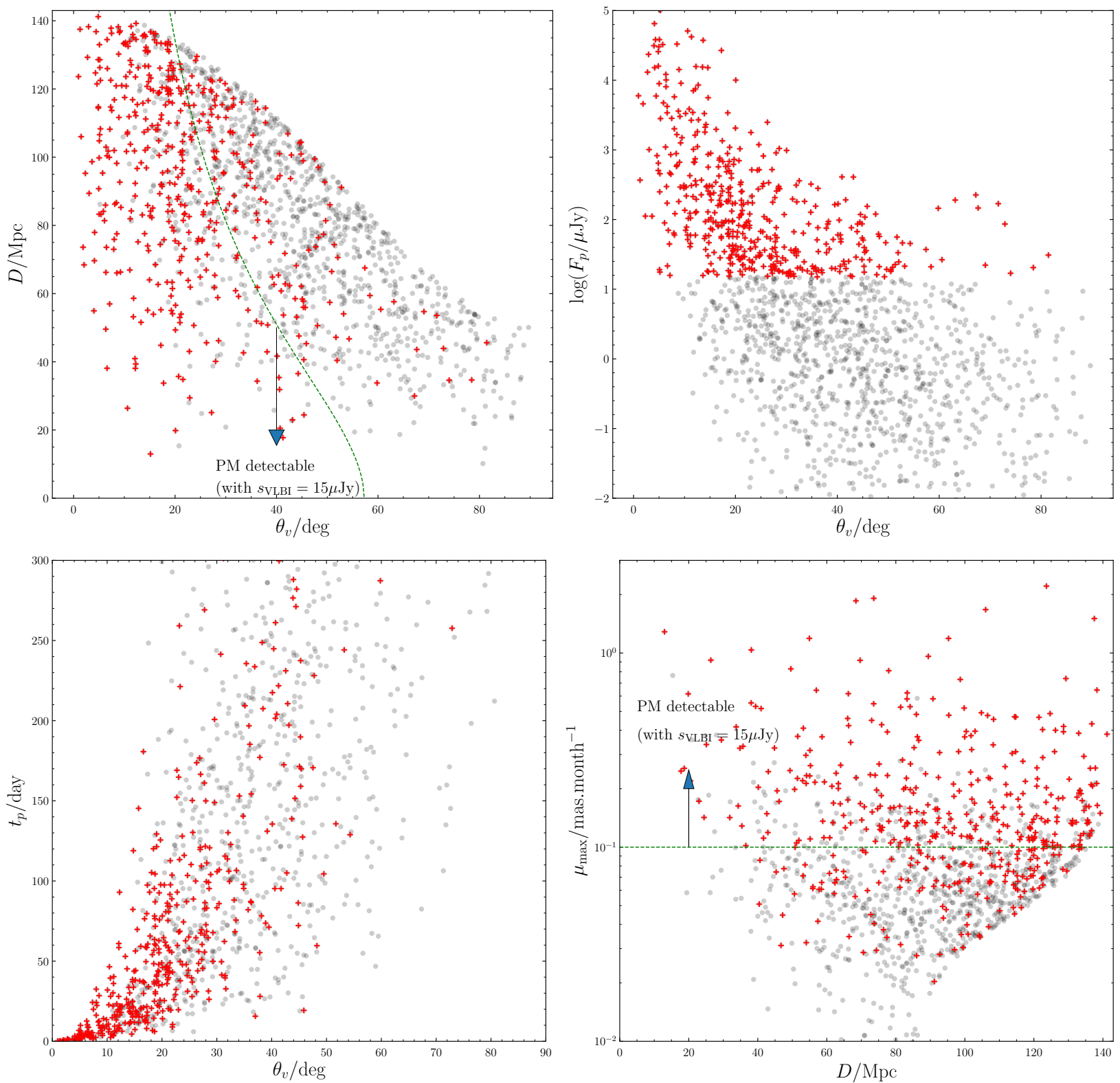

Fig. 7. Predicted samples of GW-detected (dots) and jointly detected (crosses) mergers in planes of various pairs of observables, for O3-VLA configuration. Upper left: distance and viewing angle, and maximum distance at which the proper motion can be measured $\left(D_{\max }\left(\theta_{\mathrm{v}}\right)\right.$, see Eq. $(18)$, green dotted line). Upper right: peak flux and viewing angle. Lower left: time of radio afterglow peak (assuming lateral expansion of jet) and viewing angle. Lower right: maximum remnant proper motion and distance, and limiting proper motion $\mu_{\text {lim }}$ (green dotted line).

\subsection{Impact of the population model uncertainties on the predicted population}

\subsubsection{Jet energy distribution and opening angle}

Here we consider alternatives to our fiducial population model. With a distribution of jet energy that increases below the break (i.e., taking $\alpha_{1}<0$ in Eq. (11)) as would be obtained from the short GRB luminosity function of Wanderman \& Piran (2015) $\left(\alpha_{1}=-0.9\right)$ and still assuming the same standard values for the duration and efficiency, the fraction of events detectable with the VLA would typically be three times smaller: $15 \%$ for $\mathrm{O} 2$ and $10 \%$ for $\mathrm{O} 3$ compared to $43 \%$ and $31 \%$ with $\alpha_{1}=0.5$. The viewing angle and peak times would also be strongly affected, showing mean $\theta_{v}$ 's for $\mathrm{O} 2$ (resp. O3) at only $17^{\circ}$ (resp. $14^{\circ}$ ), 98\% (resp. 99\%) of afterglows peaking before 150 days in the jet expansion hypothesis, and 90\% (resp. 93\%) without lateral expansion.
These results were obtained assuming a typical value $f_{\gamma}=$ $20 \%$ for gamma-ray efficiency but one cannot exclude that $f_{\gamma}$ may vary from from less than $1 \%$ to more than $20 \%$. Estimates of $f_{\gamma}$ from afterglow fitting have indeed been found to cover a large interval (e.g., Lloyd-Ronning \& Zhang 2004; Zhang et al. 2007). Thus, the three orders of magnitude in isotropic gamma-ray luminosity could partially result from differences in efficiency, the jet isotropic kinetic energy being restricted to a smaller interval. Figure 9 shows the effect of increasing the minimum kinetic energy $E_{\min }$ from $10^{50}$ to $10^{52} \mathrm{erg}$ in the energy function deduced from the short GRB luminosity function of Wanderman \& Piran (2015) on the detected fraction, the mean viewing angle and the fraction of afterglows to peak before 150 days. As could be expected, when the minimum kinetic energy was increased, the results came closer to those of the fiducial model in which the energy distribution function decreases below the peak. 

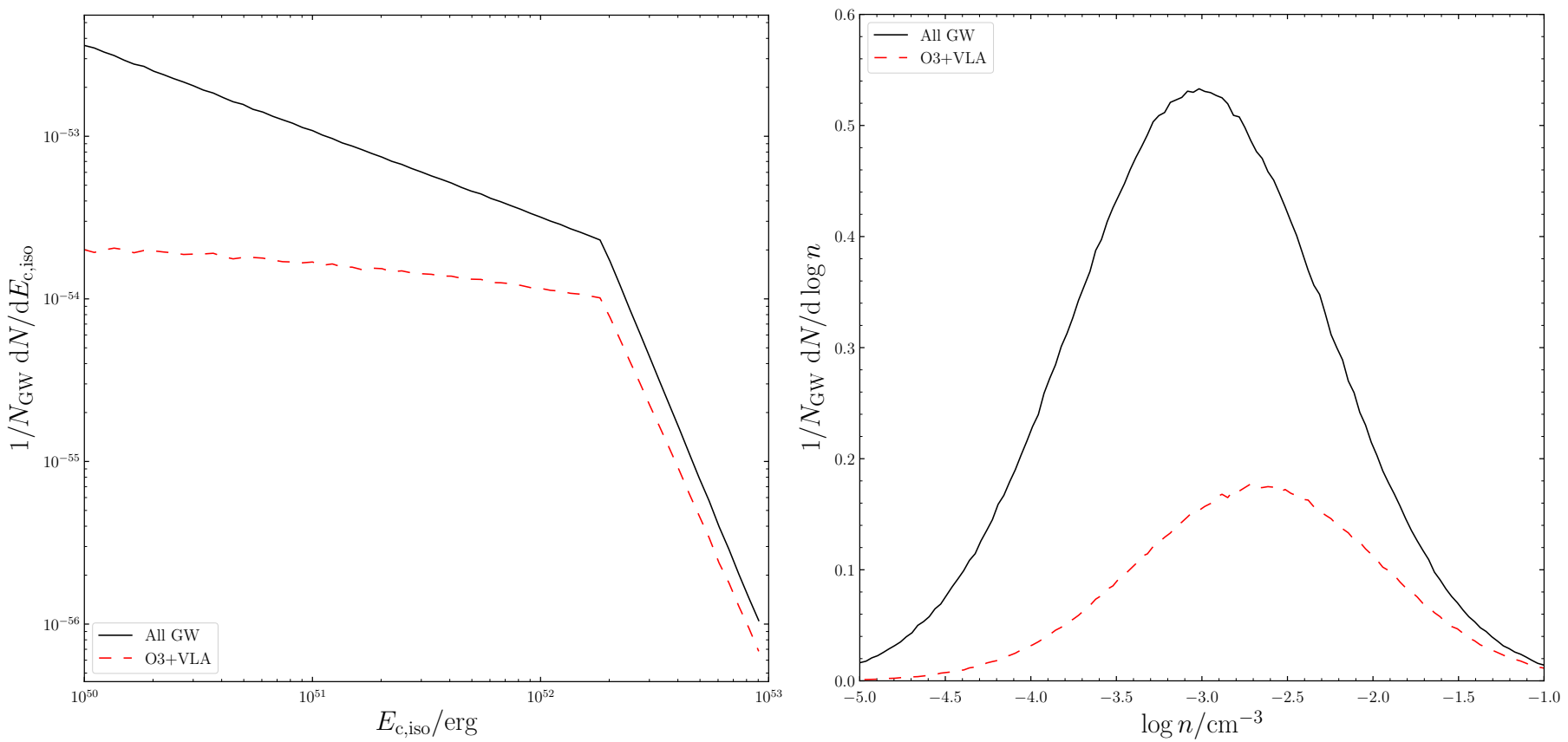

Fig. 8. Differential distribution of core jet kinetic energy (left) and external medium density (right) of entire GW population (full) and jointly detected events (dashed), in O3-VLA configuration. Distributions are normalized to fraction of jointly detected events among all GW-detected events, see text for details.
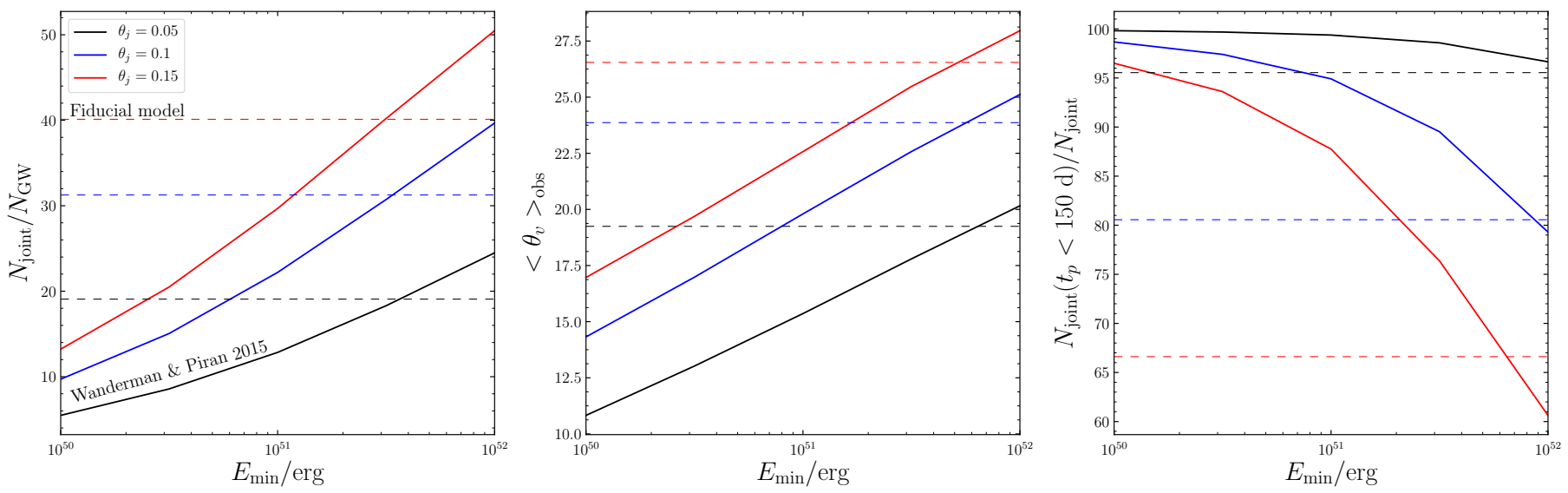

Fig. 9. Left: fraction of joint events among GW events assuming short GRB luminosity function from Wanderman \& Piran (2015) for different values of minimum kinetic energy $E_{\mathrm{m}}$ (full lines), and for our fiducial model (dashed lines), in O3-VLA configuration. Results have been plotted for different values of the jet opening angle $\theta_{\mathrm{j}}=0.05,0.1$ and $0.15 \mathrm{rad}$ (red, black and blue lines respectively). Middle: mean viewing angle (same color coding). Right: fraction of joint events with peaks earlier than 150 days post-merger, with hypothesis of jet lateral expansion, same color coding.

We also show in Fig. 9 the effect of changing the typical value of the jet opening angle from 0.1 to 0.05 or $0.15 \mathrm{rad}$. When $\theta_{\mathrm{j}}=0.05$ (resp. 0.15), the detected fraction decreases (resp. increases) to $19 \%$ (resp. $40 \%$ ), the mean viewing angle decreases (resp. increases) by $4^{\circ}$, and the peak of the light curve generally occurs later (resp. earlier).

\subsubsection{External density distribution and microphysics}

The afterglow peak flux depends greatly on the density of the circum-merger medium, as can be seen from Eq. (6). Therefore, the ability to detect the afterglows of a population of mergers depends strongly on the density of the media hosting the binaries upon merger. This is illustrated in Table 4, where we give this fraction for different external density distribution central values. These figures were calculated using the semi-analytical model (Eq. (4)), taking into account the full synchrotron spectrum including self-absorption.

If there is a significant population of fast-merging neutron star binaries, as proposed by Matteucci et al. (2014), Hotokezaka et al. (2015), Vangioni et al. (2016), Beniamini \& Piran (2016), for example, these should merge in higher density environments, producing brighter afterglows and offering the possibility to notably contribute to the observed population even if they are intrinsically fewer in number. With a density distribution centered at $n=10 \mathrm{~cm}^{-3}$, and the other parameters taking their fiducial values, $99 \%$ of GW events produce detectable radio afterglows in the O3-VLA configuration. 
Table 4. Fraction of joint events among GW events for different intrinsic density distribution central values, for the O3-VLA detector configuration, as calculated with the semi-analytical model (Eq. (4)).

\begin{tabular}{ll}
\hline \hline$n_{0} / \mathrm{cm}^{-3}$ & $N_{\text {joint }} / N_{\mathrm{GW}}$ \\
\hline $10^{-4}$ & $22 \%$ \\
$10^{-3}$ & $40 \%$ \\
$10^{-2}$ & $63 \%$ \\
0.1 & $83 \%$ \\
1 & $94 \%$ \\
10 & $97 \%$ \\
\hline
\end{tabular}

The microphysics parameter $\epsilon_{\mathrm{B}}$ enters in Eq. (6) with the same power as the external density. Moreover, our fiducial choices for the distribution of these two quantities are nearly the same (with simply a limit in range for $\epsilon_{\mathrm{B}}$ ) so that changing its central value affects the detected fraction in the same way.

\section{Discussion and conclusion}

We studied the population of binary neutron star mergers to be observed jointly through GW and their radio afterglows in future multi-messenger observing campaigns. To do so, we have assumed a likely population of mergers inspired by prior short GRB science, and simulated the GW and jet-dominated radio afterglow emissions from these. We have made predictions on the rates of such future events, and on how the observables from these events are expected to be distributed among themselves.

In the case of the ongoing $\mathrm{O} 3$ run of the LVC, and assuming our fiducial set of parameters (see Table 1), we predict that $\sim 30 \%$ of GW events should have a radio afterglow detectable by the VLA. These joint events should have mean viewing angles of $\sim 24^{\circ}$, and should peak earlier than 150 days post-merger in $\sim 80 \%$ of cases, assuming lateral expansion of the jet.

As illustrated in the 170817 event, the lateral material may also contribute to the peak flux of the radio afterglow. Figure 1 (right) shows that this may increase the peak flux by a factor of up to 1.5. In this case, our $\mathrm{O} 3$ predictions are revised to a fraction of $36 \%$ of events with detectable afterglow and a similar mean viewing angle of $25^{\circ}$.

\subsection{Detecting the detectable events}

Throughout this work, we have applied a threshold on the GW $\mathrm{S} / \mathrm{N}$ and radio afterglow peak flux to label an event as jointly detected. Thus our study concerns the class of detectable events. The fraction of these detectable events that can actually be detected heavily depends on the accuracy of the localization, which is related to the size of the localization skymap provided by the GW network, the possible detection of an associated GRB, and the efficiency of the search for a kilonova in terms of covered sky area and limiting magnitude.

Assuming that all neutron star mergers produce a kilonova, and even taking into account a probable anisotropy of the emission, it is expected that $100 \%$ of kilonovae are detectable during O3 for a limiting magnitude of 21 (Duque et al. 2019; Mochkovitch et al. in prep.). However, the search for the kilonova becomes complicated due to the large localization skymaps of GW events. These error boxes can be improved in the case when an associated GRB is being detected, which should, however, tend to remain rare (see Sect. 4.1.2 and Beniamini et al
2019). During O3, existing facilities with large fields of view in the optical band are experiencing difficulty in covering $100 \%$ of $\gtrsim 1000 \mathrm{deg}^{2}$ skymaps with a deep limiting magnitude, especially in the southern sky. Instruments such as the Zwicky Transient Facility (Bellm et al. 2019) cover the northern sky down to magnitude $r \sim 20.5$.

On the other hand, when the LIGO/Virgo instruments will have achieved their design sensitivities, it is expected that one or two new interferometers will join the GW network: KAGRA (Kagra Collaboration 2019) and Ligo-India (Iyer 2015). This should lead to a significant improvement in localization. In addition, the deep coverage of even large error boxes will be made possible by new facilities such as the Large Synoptic Survey Telescope (Ivezic et al. 2008). Nevertheless, there are other difficulties related to the search for the kilonova: kilonova/host galaxy contrast at large distances, possible large offsets, availability of a photometric and spectroscopic follow-up of the candidates, etc. In this context, it is clear that the efficiency of the search for kilonovae will always remain below $100 \%$, but should increase with time and may potentially be high in the design configuration of the GW network.

Even assuming the localization has been acquired thanks to the detection of a kilonova, a continuous monitoring of the remnant up to $\sim 150$ days may be necessary to detect the peak of the radio afterglow, in the case of marginally detectable events. Even then, only events with peaks somewhat larger than the radio threshold should yield observations of astrophysical interest because extended observations of the rise, peak and decay of the afterglow are necessary to resolve the structure and dynamics (e.g., expansion) of the ultra-relativistic jet, as illustrated by the case of GRB170817A.

As also illustrated in the case of GRB170817A, VLBI measurements are instrumental in assessing the presence and resolution of the jet structure. Therefore, an even more restrictive criterion for full event characterization might be the detectability of the remnant proper motion. As we demonstrated in Sect. 4.1.4, this would primarily decrease the fraction of events, especially in the context of design GW detectors.

\subsection{Constraining the BNS population and short GRBs with radio afterglows}

We have studied the sensitivity of the expected distributions of afterglow observables to the population parameters, as well as the radio and $\mathrm{GW}$ detector configurations. In particular, we have shown the uncertainties on the rates and typical viewing angles of these events which have been inherited from the uncertainties on the luminosity function of short GRBs and of the density of the media hosting the mergers.

In Sects. 4.1.1 and 4.1.2 we discussed the combined influence of increasing the radio and GW detector sensitivity on the rate and viewing angle of the events. At this stage, both improvements would be beneficial as the predicted rate in the current O3-VLA configuration is well below $50 \%$ of joint events among GW events.

As the GW horizon increases, more events will be seen onaxis. In the case of an opening angle of $0.1 \mathrm{rad}$ and in the likely short-term evolution of the detector configuration from O3-VLA (current) to design-VLA (early 2020s), we predict that 5\% to $10 \%$ of events should be seen on-axis, increasing the probability of a GRB counterpart in addition to the GW and afterglow. This figure is fully consistent with that announced in Beniamini et al. (2019) for events with a GW+GRB association. In fact, as a $\mathrm{GW}+$ classical GRB association is equivalent to an on-axis event, 
one may measure the opening angle of typical GRB jets by considering the ratio of $\mathrm{GW}$ events with afterglow counterpart only to events with both afterglow and GRB, thus exploiting the new possibility of observing afterglows without detecting the GRB.

More generally, a population of observed events corresponds to an intrinsic population of mergers. Thus, a comparison of a statistical number of joint event observations to our predicted distributions is a means of measuring fundamental parameters of the population of mergers, and of constraining GRB quantities, such as their energy function.

Finally, future facilities such as the SKA may bring detections of orphan radio afterglows through deep radio surveys. These would not be subject to the GW criterion and may probe another sub-population of mergers, bringing yet another class of constraints upon these phenomena.

In conclusion, regardless of the evolution of $\mathrm{GW}$ and radio detectors, multi-wavelength afterglows will remain instrumental in the study of binary neutron star mergers as windows on both their environment and their role as progenitors of short GRBs. They will bring invaluable insight at the level of the population of mergers and on an event-to-event basis, provided the sources may be accurately localized in the sky.

Acknowledgements. The authors acknowledge financial support from the Centre National d'Études Spatiales (CNES), and thank Paz Beniamini for useful discussions.

\section{References}

Abbott, B. P., Abbott, R., Abbott, T. D., et al. 2017, Phys. Rev. Lett., 119, 161101 Abbott, B. P., Abbott, R., Abbott, T. D., et al. 2018, Liv. Rev. Rel., 21, 3 Abbott, B. P., Abbott, R., Abbott, T. D., et al. 2019, Phys. Rev. X, 9, 011001 Alexander, K. D., Margutti, R., Blanchard, P. K., et al. 2018, ApJ, 863, L18 Bellm, E. C., Kulkarni, S. R., Graham, M. J., et al. 2019, PASP, 131, 018002 Beniamini, P., \& Piran, T. 2016, MNRAS, 456, 4089

Beniamini, P., \& van der Horst, A. J. 2017, MNRAS, 472, 3161

Beniamini, P., Nava, L., \& Piran, T. 2016, MNRAS, 461, 51

Beniamini, P., Petropoulou, M., Barniol Duran, R., \& Giannios, D. 2019, MNRAS, 483, 840

Berger, E. 2014, Annu. Rev. Astron. Astrophys., 52, 43

Braun, R. 2014, SKA Technical Document, SKA-TEL.SCI-SKO-SRQ-001

Cantiello, M., Jensen, J. B., Blakeslee, J. P., et al. 2018, ApJ, 854, L31

Church, R. P., Levan, A. J., Davies, M. B., \& Tanvir, N. 2011, MNRAS, 413, 2004

Coulter, D., Foley, R., Kilpatrick, C., et al. 2018, APS April Meeting Abstracts, 2018, D16.002

Cowperthwaite, P. S., Berger, E., Villar, V. A., et al. 2017, ApJ, 848, L17

D'Avanzo, P., Salvaterra, R., Bernardini, M. G., et al. 2014, MNRAS, 442, 2342

D’Avanzo, P., Campana, S., Salafia, O. S., et al. 2018, A\&A, 613, L1

Dobie, D., Kaplan, D. L., Murphy, T., et al. 2018, ApJ, 858, L15

Duffell, P. C., Quataert, E., Kasen, D., \& Klion, H. 2018, ApJ, 866, 3

Duque, R., Mochkovitch, R., \& Daigne, F. 2019, Proceedings of Science

Evans, P. A., Cenko, S. B., Kennea, J. A., et al. 2017, Science, 358, 1565

Finn, L. S., \& Chernoff, D. F. 1993, Phys. Rev. D, 47, 2198

Fong, W., Berger, E., \& Fox, D. B. 2010, ApJ, 708, 9

Fong, W., Berger, E., Margutti, R., \& Zauderer, B. A. 2015, ApJ, 815, 102

Ghirlanda, G., Salafia, O. S., Pescalli, A., et al. 2016, A\&A, 594, A84

Ghirlanda, G., Salafia, O. S., Paragi, Z., et al. 2019, Science, 363, 968

Gill, R., \& Granot, J. 2018, MNRAS, 478, 4128
Goldstein, A., Veres, P., Burns, E., et al. 2017, ApJ, 848, L14 Gottlieb, O., Nakar, E., \& Piran, T. 2018, MNRAS, 473, 576 Gottlieb, O., Nakar, E., \& Piran, T. 2019, MNRAS, 488, 2405 Granot, J., Panaitescu, A., Kumar, P., \& Woosley, S. E. 2002, ApJ, 570, L61

Haggard, D., Nynka, M., Ruan, J. J., et al. 2017, ApJ, 848, L25 Hallinan, G., Corsi, A., Mooley, K. P., et al. 2017, Science, 358, 1579 Hotokezaka, K., Piran, T., \& Paul, M. 2015, Nat. Phys., 11, 1042 Hotokezaka, K., Kiuchi, K., Shibata, M., Nakar, E., \& Piran, T. 2018, ApJ, 867, 95

Ivezic, Z., Axelrod, T., Brandt, W. N., et al. 2008, Serb. Astron. J., 176, 1

Iyer, B. 2015, APS April Meeting Abstracts, 2015, J13.001

Kagra Collaboration (Akutsu, T., et al.) 2019, Nat. Astron., 3, 35

Kasliwal, M. M., Nakar, E., Singer, L. P., et al. 2017, Science, 358, 1559

Lamb, G. P., \& Kobayashi, S. 2017, MNRAS, 472, 4953

Lazzati, D., Deich, A., Morsony, B. J., \& Workman, J. C. 2017, MNRAS, 471, 1652

Ligo Scientific Collaboration \& VIRGO Collaboration 2019a, GRB Coordinates Network, 24168, 1

Ligo Scientific Collaboration \& VIRGO Collaboration 2019b, GRB Coordinates Network, 24237, 1

Lloyd-Ronning, N. M., \& Zhang, B. 2004, ApJ, 613, 477

Lyman, J. D., Lamb, G. P., Levan, A. J., et al. 2018, Nat. Astron., 2, 751

Margutti, R., Berger, E., Fong, W., et al. 2017, ApJ, 848, L20

Margutti, R., Alexander, K. D., Xie, X., et al. 2018, ApJ, 856, L18

Matteucci, F., Romano, D., Arcones, A., Korobkin, O., \& Rosswog, S. 2014, MNRAS, 438, 2177

Mooley, K. P., Frail, D. A., Dobie, D., et al. 2018a, ApJ, 868, L11

Mooley, K. P., Nakar, E., Hotokezaka, K., et al. 2018b, Nature, 554, 207

Mooley, K. P., Deller, A. T., Gottlieb, O., et al. 2018c, Nature, 561, 355

Nakar, E. 2007, Phys. Rep., 442, 166

Nakar, E., Piran, T., \& Granot, J. 2002, ApJ, 579, 699

Nakar, E., Gottlieb, O., Piran, T., Kasliwal, M. M., \& Hallinan, G. 2018, ApJ, 867,18

Palmese, A., Hartley, W., Tarsitano, F., et al. 2017, ApJ, 849, L34

Panaitescu, A., \& Kumar, P. 2000, ApJ, 543, 66

Panaitescu, A., \& Kumar, P. 2001, ApJ, 560, L49

Pian, E., D'Avanzo, P., Benetti, S., et al. 2017, Nature, 551, 67

Resmi, L., Schulze, S., Ishwara-Chandra, C. H., et al. 2018, ApJ, 867, 57

Saleem, M., Pai, A., Misra, K., Resmi, L., \& Arun, K. G. 2018a, MNRAS, 475, 699

Saleem, M., Resmi, L., Misra, K., Pai, A., \& Arun, K. G. 2018b, MNRAS, 474, 5340

Santana, R., Barniol Duran, R., \& Kumar, P. 2014, ApJ, 785, 29

Sari, R., Piran, T., \& Narayan, R. 1998, ApJ, 497, L17

Savchenko, V., Ferrigno, C., Kuulkers, E., et al. 2017, ApJ, 848, L15

Selina, R. J., Murphy, E. J., McKinnon, M., et al. 2018, in Ground-based and Airborne Telescopes VII, SPIE Conf. Ser., 10700, 1070010

Sironi, L., \& Spitkovsky, A. 2011, ApJ, 726, 75

Sironi, L., Keshet, U., \& Lemoine, M. 2015, Space Sci. Rev., 191, 519

Smartt, S. J., Chen, T.-W., Jerkstrand, A., et al. 2017, Nature, 551, 75

Tanvir, N. R., Levan, A. J., González-Fernández, C., et al. 2017, ApJ, 848, L27

Troja, E., King, A. R., O’Brien, P. T., Lyons, N., \& Cusumano, G. 2008, MNRAS, 385, L10

Troja, E., Piro, L., van Eerten, H., et al. 2017, Nature, 551, 71

Troja, E., Piro, L., Ryan, G., et al. 2018, MNRAS, 478, L18

Valenti, S., Sand, D. J., Yang, S., et al. 2017, ApJ, 848, L24

van Eerten, E. T. H., Ryan, G., Ricci, R., et al. 2019, MNRAS, 489, 1919

Vangioni, E., Goriely, S., Daigne, F., François, P., \& Belczynski, K. 2016, MNRAS, 455, 17

Wanderman, D., \& Piran, T. 2015, MNRAS, 448, 3026

Wijers, R. A. M. J., \& Galama, T. J. 1999, ApJ, 523, 177

Xie, X., Zrake, J., \& MacFadyen, A. 2018, ApJ, 863, 58

Zhang, B., Liang, E., Page, K. L., et al. 2007, ApJ, 655, 989 